\title{
Nod2-Nodosome in a Cell-Free System: Implications in Pathogenesis and Drug Discovery for Blau Syndrome and Early-Onset Sarcoidosis
}

\author{
Tomoyuki Iwasaki, ${ }^{1}$ Naoe Kaneko, ${ }^{1}$ Yuki Ito, ${ }^{1}$ \\ Hiroyuki Takeda, ${ }^{2}$ Tatsuya Sawasaki, ${ }^{2}$ Toshio Heike, ${ }^{3}$ Kiyoshi Migita, \\ Kazunaga Agematsu, ${ }^{5}$ Atsushi Kawakami, ${ }^{6}$ Shinnosuke Morikawa, ${ }^{1}$ \\ Sho Mokuda, ${ }^{1}$ Mie Kurata, ${ }^{1}$ and Junya Masumoto ${ }^{1}$ \\ ${ }^{1}$ Department of Pathology, Ehime University Proteo-Science Center and Graduate School of Medicine, Shitsukawa 454, \\ Toon, Ehime 791-0295, Japan \\ ${ }^{2}$ Division of Cell-Free Sciences, Ehime University Proteo-Science Center, Bunkyocho 3, Matsuyama, Ehime 790-8577, Japan \\ ${ }^{3}$ Department of Pediatrics, Kyoto University Graduate School of Medicine, Shogoin Kawaramachi 54, Kyoto 606-8507, Japan \\ ${ }^{4}$ Clinical Research Center, Nagasaki Medical Center, Kubara 2-1001-1, Omura, Nagasaki 856-8562, Japan \\ ${ }^{5}$ Department of Infection and Host Defense, Shinshu University Graduate School of Medicine, Asahi 3-1-1, Matsumoto, \\ Nagano 390-8621, Japan \\ ${ }^{6}$ Unit of Translational Medicine, Department of Immunology and Rheumatology, Nagasaki University Graduate School of \\ Biomedical Sciences, Medicine, Sakamoto 1-7-1, Nagasaki 852-8501, Japan \\ Correspondence should be addressed to Junya Masumoto; masumoto@m.ehime-u.ac.jp
}

Received 11 March 2016; Accepted 23 May 2016

Academic Editor: Rolando Cimaz

Copyright (C) 2016 Tomoyuki Iwasaki et al. This is an open access article distributed under the Creative Commons Attribution License, which permits unrestricted use, distribution, and reproduction in any medium, provided the original work is properly cited.

\begin{abstract}
Nucleotide-binding oligomerization domain-containing protein (Nod) 2 is an intracellular pattern recognition receptor, which recognizes muramyl dipeptide (N-Acetylmuramyl-L-Alanyl-D-Isoglutamine: MDP), a bacterial peptidoglycan component, and makes a NF- $\kappa \mathrm{B}$-activating complex called nodosome with adaptor protein RICK (RIP2/RIPK2). Nod2 mutants are associated with the autoinflammatory diseases, Blau syndrome (BS)/early-onset sarcoidosis (EOS). For drug discovery of BS/EOS, we tried to develop Nod2-nodosome in a cell-free system. FLAG-tagged RICK, biotinylated-Nod2, and BS/EOS-associated Nod2 mutants were synthesized, and proximity signals between FLAG-tagged and biotinylated proteins were detected by amplified luminescent proximity homogeneous assay (ALPHA). Upon incubation with MDP, the ALPHA signal of interaction between Nod2-WT and RICK was increased in a dose-dependent manner. The ALPHA signal of interaction between RICK and the BS/EOS-associated Nod2 mutants was more significantly increased than Nod2-WT. Notably, the ALPHA signal between Nod2-WT and RICK was increased upon incubation with MDP, but not when incubated with the same concentrations, L-alanine, D-isoglutamic acid, or the MDP-D-isoform. Thus, we successfully developed Nod2-nodosome in a cell-free system reflecting its function in vivo, and it can be useful for screening Nod2-nodosome-targeted therapeutic molecules for BS/EOS and granulomatous inflammatory diseases.
\end{abstract}

\section{Introduction}

Nucleotide-binding oligomerization domain-containing protein (Nod) 2 is a nuclear factor- (NF-) $\kappa$ B-activating intracellular pattern recognition receptor, which was identified as a susceptibility gene product of Crohn's disease, an inflammatory bowel disease [1-3].
Nod2 was reported to be oligomerized with adaptor protein RICK (RIP2/RIPK2) and IKK complexes, which can activate NF- $\kappa$ B by muramyl dipeptide (N-AcetylmuramylL-Alanyl-D-Isoglutamine: MDP), one of the components of bacterial cell-wall peptidoglycan, and is utilized as an immune-stimulatory adjuvant for vaccination and for developing antibodies [4-8]. 
TABLE 1: Oligonucleotide sequences for plasmid construction.

\begin{tabular}{|c|c|}
\hline Primer name & Primer sequence \\
\hline S1-Nod2_F & $5^{\prime}$-CCACCCACCACCACCAATGGGGGAAGAGGGTGGTTCAG- $3^{\prime}$ \\
\hline Nod2-T1(F)_R & $5^{\prime}$-TCCAGCACTAGCTCCAGAAAGCAAGAGTCTGGTGTCCCT-3' \\
\hline Nod2-T1(CARDs)_R & $5^{\prime}$-TCCAGCACTAGCTCCAGACTTGCATGTGGCAGCTTCCA-3' \\
\hline S1-RICK_F & $5^{\prime}$-CCACCCACCACCACCAATGAACGGGGAGGCCATCTG- $3^{\prime}$ \\
\hline RICK-T1(F)_R & $5^{\prime}$-TCCAGCACTAGCTCCAGACATGCTTTTATTTTGAAGTAAATTTA-3' \\
\hline S1-RICK(CARD)_F & $5^{\prime}$-CCACCCACCACCACCAATGCTGCAGCCTGGTATAGCCC- $3^{\prime}$ \\
\hline attB1-S1 & $5^{\prime}$-GGGGACAAGTTTGTACAAAAAAGCAGGCTTCCACCCACCACCACCAATG-3' \\
\hline attB2-T1 & 5'-GGGGACCACTTTGTACAAGAAAGCTGGGTCTCCAGCACTAGCTCCAGA-3' \\
\hline Nod2-R334W_F & 5'-TTCCCATTCAGCTGCTGGCAGCTGCAGTGC-3' \\
\hline Nod2-R334W_R & $5^{\prime}$-GCACTGCAGCTGCCAGCAGCTGAATGGGAA-3' \\
\hline Nod2-N670K_F & $5^{\prime}$-GCCGAGCCGCACAAACTTCAGATCACAGCA-3' \\
\hline Nod2-N670K_R & $5^{\prime}$-TGCTGTGATCTGAAGTTTGTGCGGCTCGGC-3' \\
\hline
\end{tabular}

Underline indicates $\mathrm{S} 1$ or $\mathrm{T} 1$ sequence.

Bold indicates Kozak consensus sequence.

Italic indicates mutation codons for specific amino acids.

It was also discovered that an autoinflammatory disease, Blau syndrome (BS)/early-onset sarcoidosis (EOS), was caused by a point mutation of Nod2, encoding a constitutively active form, resulting in NF- $\kappa \mathrm{B}$ activation [9-11]. $\mathrm{BS} / \mathrm{EOS}$ is a systemic granulomatous disease, and patients with BS/EOS have nonnecrotizing granulomas of the skin, eyes, and joints [12]. Other granulomatous inflammatory diseases were similarly reported to be associated with Nod2 $[13,14]$. Therefore, Nod2 has been thought to be involved in granulomatous disorders [15] and may be an attractive drug target for treatment of these chronic inflammatory granulomatous diseases.

Thus, we aimed to develop a reconstituted protein-protein interaction assay system between wild-type Nod2 and the BS/EOS-associated mutants of Nod 2 and RICK in a cell-free system, further called the reconstituted Nod2-nodosome in a cell-free system [16].

\section{Materials and Methods}

2.1. Plasmid Construction, Site-Specific Mutagenesis for the BS/EOS-Associated Mutant Nod2, and Protein Synthesis. The cDNA of human Nod2 and RICK were derived from pcDNA3-Nod2 and pcDNA3-RICK, kindly provided by Dr. G Núñez (accession number: AF178930 and AF027706). Each open reading frame, without stop codons, was modified in a two-step polymerase chain reaction (PCR). Briefly, the initial PCR product for Nod2-WT was amplified using the primers: forward S1-Nod2_F: $5^{\prime}$-CCACCCACCACCACCAATGGGGGAAGAGGGTGGTTCAG-3' and reverse Nod2-T1(F)_R: 5'-TCCAGCACTAGCTCCAGAAAGCAAGAGTCTGGTGTCCCT- $3^{\prime}$. The initial PCR product for Nod2-CARD1+CARD2 (CARDs) was amplified using the primers: forward S1-Nod2_F: $5^{\prime}$-CCACCCACCACCACCAATGGGGGAAGAGGGTGGTTCAG-3' and reverse Nod2-T1(CARDs)_R: 5' -TCCAGCACTAGCTCCAGACTTGCATGTGGCAGCTTCCA-3'.

The initial PCR product for RICK-WT was amplified using the primers: forward S1-RICK_F: $5^{\prime}$-CCACCCACCACCACCAATGAACGGGGAGGCCATCTG- $3^{\prime}$ and reverse RICK-T1(F)_R: 5'-TCCAGCACTAGCTCCAGACATGCTTTTATTTTGAAGTAAATTTA $-3^{\prime}$. The initial PCR product for RICK-CARD was amplified using the primers: forward S1-RICK (CARD)_F: $5^{\prime}$-CCACCCACCACCACCAATGAACGGGGAGGCCATCTG- ${ }^{\prime}$ and reverse RICK-T1(F)_R: 5'-TCCAGCACTAGCTCCAGACATGCTTTTATTTTGAAGTAAATTTA-3'.

The second PCR was carried out using the following primer set: attB1-S1: $5^{\prime}$-GGGGACAAGTTTGTACAAAAAAGCAGGCTTCCACCCACCACCACCAATG-3' and attB2-T1: $5^{\prime}$-GGGGACCACTTTGTACAAGAAAGCTGGGTCTCCAGCACTAGCTCCAGA-3' with the initial PCR products as templates. These primers are shown in Table 1.

PCR-based site-specific mutagenesis for Nod2-R334W was amplified by two-step PCR using the following primer sets: forward S1-Nod2_F: 5'-CCACCCACCACCACCAATGGGGGAAGAGGGTGGTTCAG-3' and reverse Nod2R334W_R: 5'-GCACTGCAGCTGCCAGCAGCTGAATGGGAA-3', and forward Nod2-R334W_F: 5'-TTCCCATTCAGCTGCTGGCAGCTGCAGTGC- $3^{\prime}$ and reverse Nod2-T1(F)_R: 5'-TCCAGCACTAGCTCCAGAAAGCAAGAGTCTGGTGTCCCT-3' for the first-step overlapping DNA fragment set. PCR-based site-specific mutagenesis for Nod2-N670K was also amplified by two-step PCR using the following primer sets: forward S1-Nod2_F: $5^{\prime}$-CCACCCACCACCACCAATGGGGGAAGAGGGTGGTTCAG-3' and reverse Nod2-N670K_R: 5'-TGCTGTGATCTGAAGTTTGTGCGGCTCGGC-3', and forward Nod2-N670K_F: $5^{\prime}$-GCCGAGCCGCACAAACTTCAGATCACAGCA-3' and reverse Nod2-T1(F)_R: 5'-TCCAGCACTAGCTCCAGAAAGCAAGAGTCTGGTGTCCCT- $3^{\prime}$ for the first-step overlapping DNA fragment set. R334W and N670K mutatedNod2 DNA fragments were amplified by a second PCR step using the following primer set: attB1-S1: $5^{\prime}$-GGGGACAAGTTTGTACAAAAAAGCAGGCTTCCACCCACCACCACCAATG-3' and attB2-T1: 5'-GGGGACCACTTTGTACAAGAAAGCTGGGTCTCCAGCACTAGCTCCAGA-3', from the first-step overlapping DNA fragment sets as template. These oligonucleotide sequences for mutated Nod2 
are listed in Table 1. PCR products were inserted into a pDONR221 vector using Gateway ${ }^{\circledR}$ BP Clonase ${ }^{\circledR}$ II Enzyme mix (Life Technologies, Carlsbad, CA, USA) to generate entry clones. Nod2 entry clones, pDONR221-Nod2-WT, pDONR221-Nod2-R334W, pDONR221-Nod2-N670K, and pDONR221-Nod2-CARDs, were inserted into pEU-E01GW-bls-STOP for cell-free protein expression. RICK entry clones, pDONR221-RICK-WT and pDONR221-RICKCARD, were inserted into pEU-E01-FLAG-GW-STOP, using the Gateway LR Clonase ${ }^{\circledR}$ II Enzyme mix. These constructs were confirmed by sequencing (Figure 1(c)). The constructed plasmids were used to synthesize their respective proteins using the WEPRO1240 Expression Kit (Cell-free, Inc., Matsuyama, Japan) and were followed by Western blotting.

2.2. Western Blotting Analysis. A total of $1.5 \mu \mathrm{g}$ of synthetic protein was subjected to SDS-PAGE followed by Western blotting analysis. Protein detection on the blotting membranes was performed using anti-FLAG mAb M2 (SigmaAldrich, St. Louis, MO, USA), followed by peroxidaseconjugated affinity-purified $\mathrm{F}\left(\mathrm{ab}^{\prime}\right)_{2}$ fragment of goat antimouse IgG, $\mathrm{F}\left(\mathrm{ab}^{\prime}\right)_{2}$ fragment-specific (Jackson ImmunoResearch, West Grove, PA, USA) for FLAG-tagged proteins, or HRP-conjugated streptavidin (Nacalai, Kyoto, Japan) for biotinylated proteins.

2.3. Pull-Down Assay. One-microgram biotinylated-Nod2WT (Nod2-WT-Btn) and $1 \mu \mathrm{g}$ FLAG-tagged RICK-WT (FLAG-RICK-WT) lysed in $300 \mu \mathrm{L}$ NP-40 buffer [1\% Nonidet P-40, $142.5 \mathrm{mmol} / \mathrm{L} \mathrm{KCl}, 5 \mathrm{mmol} / \mathrm{L} \mathrm{MgCl}_{2}, 10 \mathrm{mmol} / \mathrm{L}$ HEPES (pH 7.6), $0.2 \mathrm{mmol} / \mathrm{L}$ phenylmethylsulfonylfluoride (PMSF), and $1 \mathrm{mmol} / \mathrm{L}$ EDTA] were precipitated with $20 \mu \mathrm{L}$ streptavidin-conjugated agarose beads (Invitrogen) with or without $5.33 \mathrm{mg} / \mathrm{mL}$ MDP (Sigma-Aldrich) and incubated for 3 hours at $4^{\circ} \mathrm{C}$. The precipitations were subjected to SDSPAGE and immunoblotting. Detection on the blotting membranes was performed using anti-FLAG mAb M2 (SigmaAldrich) or anti-Nod2 mAb 2D9 (Cayman Chemical, Ann Arbor, MI, USA).

2.4. Amplified Luminescent Proximity Homogeneous Assay. Synthesized protein-protein interactions were assessed by amplified luminescent proximity homogeneous assay (ALPHA) (PerkinElmer). A total of $100 \mathrm{ng}$ of each protein was added to ALPHA buffer $[100 \mathrm{mM}$ Tris- $\mathrm{HCl}(\mathrm{pH}$ 8.0), $0.01 \%(\mathrm{v} / \mathrm{v})$ Tween 20], $1 \mathrm{mg} / \mathrm{mL}$ BSA, $16.67 \mu \mathrm{g} / \mathrm{mL}$ streptavidin-coated donor beads (PerkinElmer, Waltham, MA, USA), $16.67 \mu \mathrm{g} / \mathrm{mL}$ protein-A-conjugated acceptor beads, and $5 \mu \mathrm{g} / \mathrm{mL}$ anti-FLAG $\mathrm{mAb} \mathrm{M} 2$ and incubated in a Shallow Well ALPHAPlate-384 (PerkinElmer) at $25^{\circ} \mathrm{C}$ for 24 hours. Fluorescence emission signals of each well were measured using an EnSpire Multimode Plate Reader (PerkinElmer).

2.5. Statistics. Results are presented as the mean and standard deviation. Levels of significance were evaluated using Student's $t$-test. A $p$ value $<0.05$ was considered statistically significant.

\section{Results}

3.1. Recombinant Nod2, RICK, and Their Mutant Proteins Were Successfully Synthesized Using a Wheat Germ Cell-Free System. The schematics of the C-terminal biotinylated full-length wild-type Nod2 (Nod2-WT-Btn), full-length R334W-mutated Nod2 (Nod2-R334W-Btn), full-length N670K-mutated Nod2 (Nod2-N670K-Btn), and tandem CARD1- and CARD2-domains-only Nod2 (Nod2-CARDs) are shown in Figure 1(a). The schematics of the N-terminal FLAG-tagged full-length RICK (FLAG-RICK-FL) and CARD-domain-only RICK (FLAG-RICK-CARD) are shown in Figure 1(b). The plasmids vectors pDONR221-Nod2WT, pDONR221-Nod2-R334W, pDONR221-Nod2-N670K, pDONR221-Nod2-CARDs, pDONR221-RICK-WT, and pDONR221-RICK-CARD were sequenced and confirmed. The proper BS/EOS-associated mutation sequences of Nod2 were confirmed (Figure 1(c)). Nod 2 and its variants, including Nod2-WT-Btn, Nod2-R334W-Btn, Nod2-N670K-Btn, and Nod2-CARDs, were successfully synthesized using the wheat germ cell-free system (Figure 1(d)). FLAG-RICK-WT and FLAG-RICK-CARD were also successfully synthesized using the wheat germ cell-free system (Figure 1(e)).

3.2. MDP-Induced Interaction between Nod2 and RICK in Nodosome in a Cell-Free System. Nod2-WT-Btn was coprecipitated with FLAG-RICK-WT when incubated with $5.33 \mathrm{mg} / \mathrm{mL}$ MDP, but not without MDP in pull-down assay (Figure 2(a)). The baseline ALPHA signal of Nod2-WTBtn and FLAG-RICK-WT interaction was approximately 2000 counts, with no stimulation (Figure 2(b)). The ALPHA signal of interaction between Nod2-WT-Btn and FLAGRICK-WT increased upon incubation with $5.33 \mathrm{mg} / \mathrm{mL}$ MDP (Sigma-Aldrich, St. Louis, MO, USA) $(p<0.01)$, whereas no increase was detected upon incubation with the same amount $(5.33 \mathrm{mg} / \mathrm{mL})$ of N-Acetylmuramyl-D-AlanylD-Isoglutamine (MDP-D-isomer) (Invivogen, San Diego, CA, USA) (Figure 2(b)). The ALPHA signal of interaction between Nod2-CARDs-Btn and FLAG-RICK-WT increased without MDP $(p<0.01)$. Furthermore, the ALPHA signal of interaction between Nod2-CARDs-Btn and FLAG-RICKCARD without MDP was significantly increased $(p<0.01)$, whereas the ALPHA signal of interaction between Nod2WT-Btn and FLAG-RICK-CARD was not increased and was similar to baseline levels (Figure 2(b)).

3.3. The MDP Degradation Products Did Not Induce Interaction between Nod2 and RICK in the Cell-Free System. Using the Nod2-nodosome in a cell-free system established above, we assessed whether MDP degradation products, such as L-alanine, D-isoglutamic acid, and $\mathrm{N}$-acetylglucosamine (GlcNAc), could induce interaction between Nod2 and RICK in the cell-free system. No increased signals were observed upon incubation with a negative control (-), MDP-D-isomer, L-alanine, D-isoglutamic acid, and GlcNAc (Figure 2(c)).

3.4. ALPHA Signal in Response to MDP Was Observed in Nod2-Nodosome in a Cell-Free System Containing the BS/EOSAssociated Mutations. To test the activity of Nod2-nodosome 


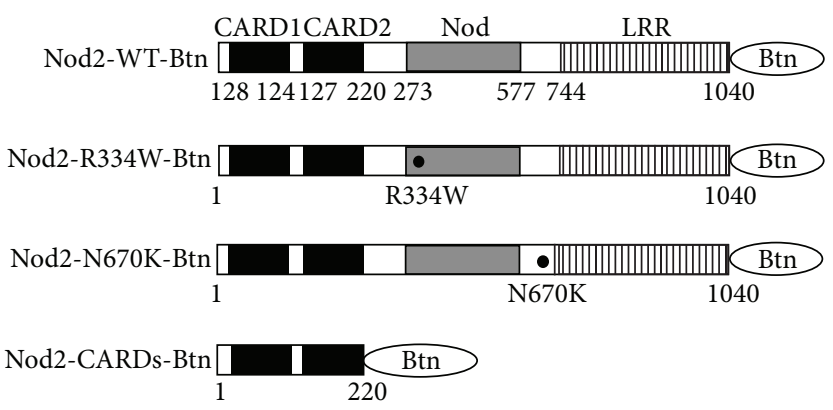

(a)

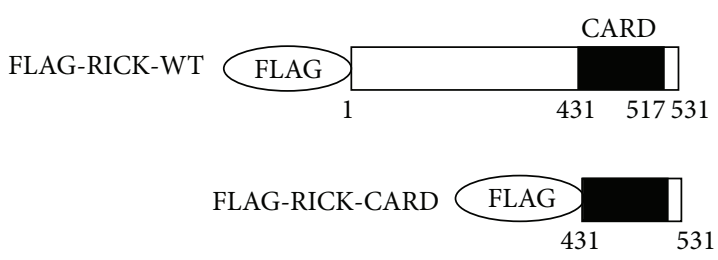

(b)
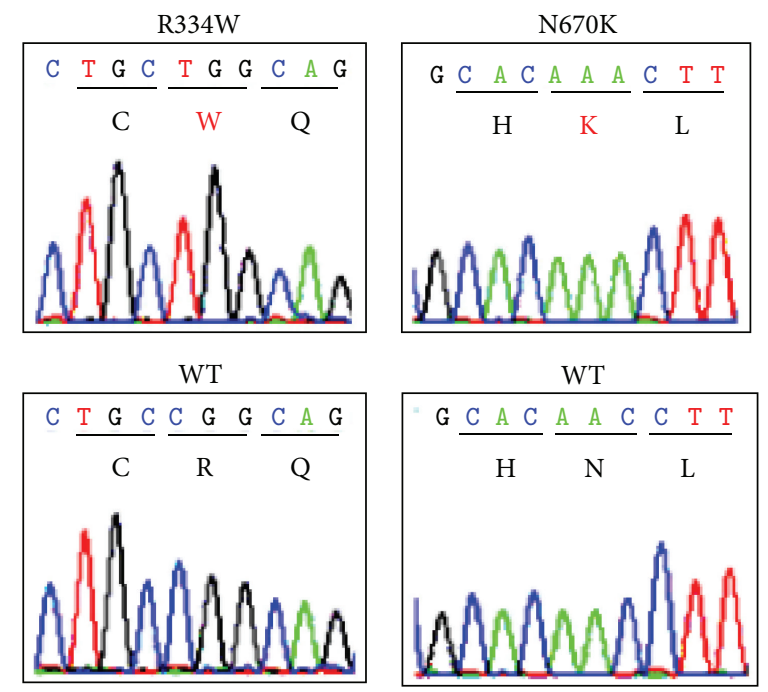

(c)

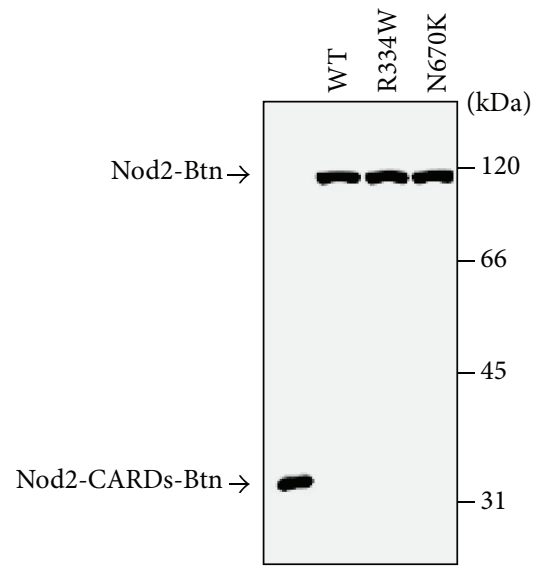

(d)

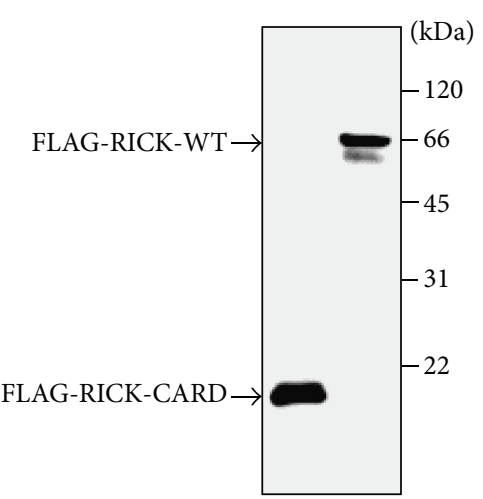

(e)

FIGURE 1: Schematic representation of Nod2 and RICK, and sequencing chromatograms of Nod2 plasmids containing the BS/EOS-associated mutations, and protein syntheses. (a) Schematic representations of biotinylated wild-type and mutant Nod2. C-terminal biotinylated fulllength wild-type Nod2 (Nod2-WT-Btn), full-length R334W-mutated Nod2 (Nod2-R334W-Btn), full-length N670K-mutated Nod2 (Nod2N670K-Btn), and tandem CARD1- and CARD2-domains-only Nod2 (Nod2-CARDs) are indicated. (b) Schematic representations of FLAGtagged wild-type and CARD-domain-only RICK. N-terminal FLAG-tagged full-length RICK (FLAG-RICK-FL) and CARD-domain-only RICK (FLAG-RICK-CARD) are indicated. The caspase recruitment domain (CARD) is indicated by black boxes. The nucleotide-binding oligomerization domain-containing protein (Nod) is indicated by grey boxes. Leucine-rich repeats are indicated by striped boxes. Amino acid sequence number and mutated amino acids are indicated under each schema. (c) Sequencing chromatograms of Nod2 and mutated-Nod2 plasmids. The wild-type and mutated-Nod2 plasmids pDONR221-Nod2-WT, pDONR221-Nod2-R334W, and pDONR221-Nod2-N670K were sequenced to confirm (from CGG to TGG corresponding to R334W in the right panel; from AAC to AAA corresponding to N670K in the left panel) mutations at the appropriate site. (d) Western blotting analysis of biotinylated Nod2 and its mutants. A total of $1.5 \mu \mathrm{g}$ of synthetic protein was subjected to SDS-PAGE followed by Western blotting. Protein detection on the membranes was performed using HRP-conjugated streptavidin. Molecular weights are indicated at right. (e) Western blotting analysis of FLAG-tagged RICK and CARD-domain-only RICK. A total of $1.5 \mu \mathrm{g}$ of synthetic protein was subjected to SDS-PAGE followed by Western blotting. Protein detection on the membranes was performed using anti-FLAG mAb M2. Molecular weights are indicated at right. 


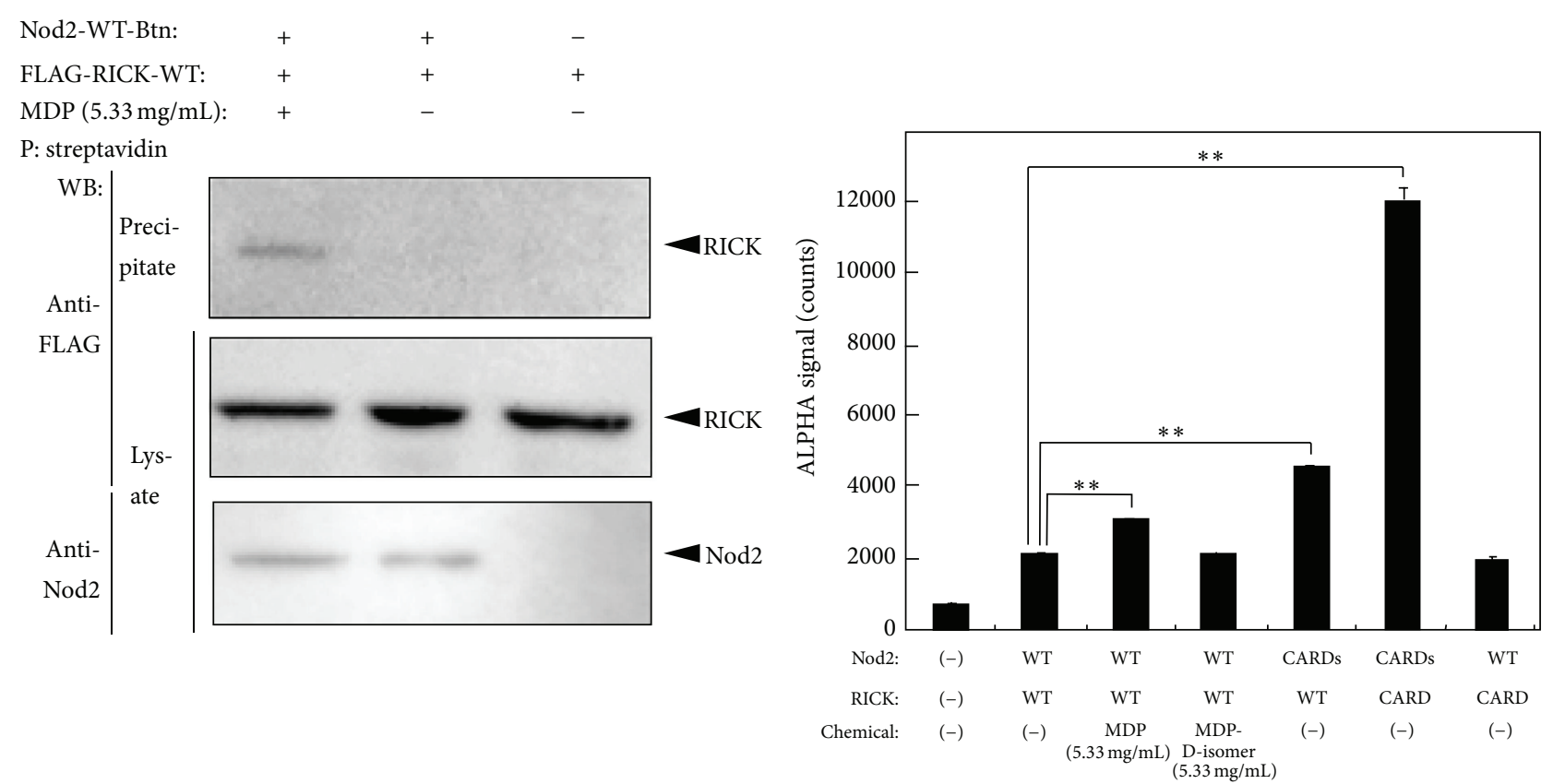

(a)

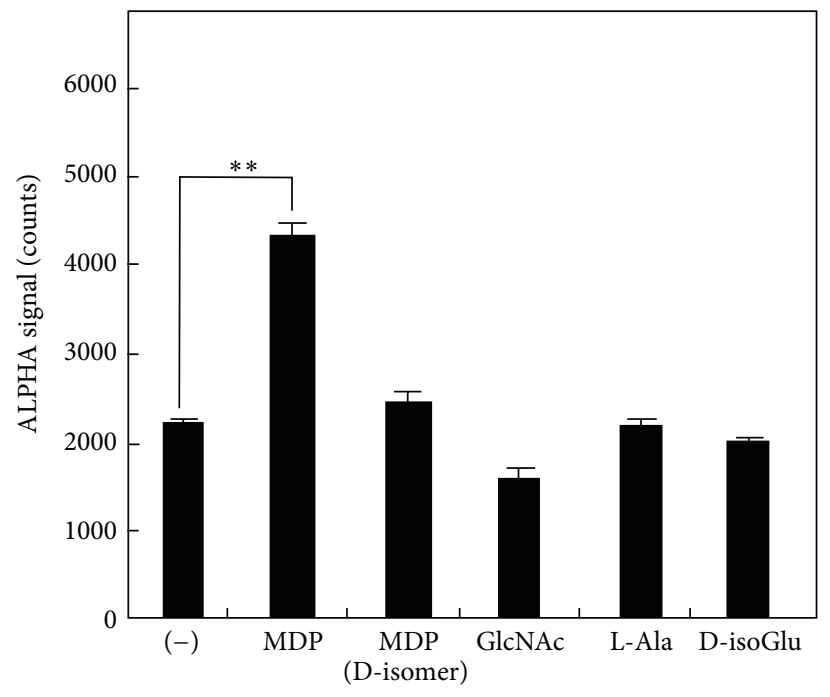

(b)

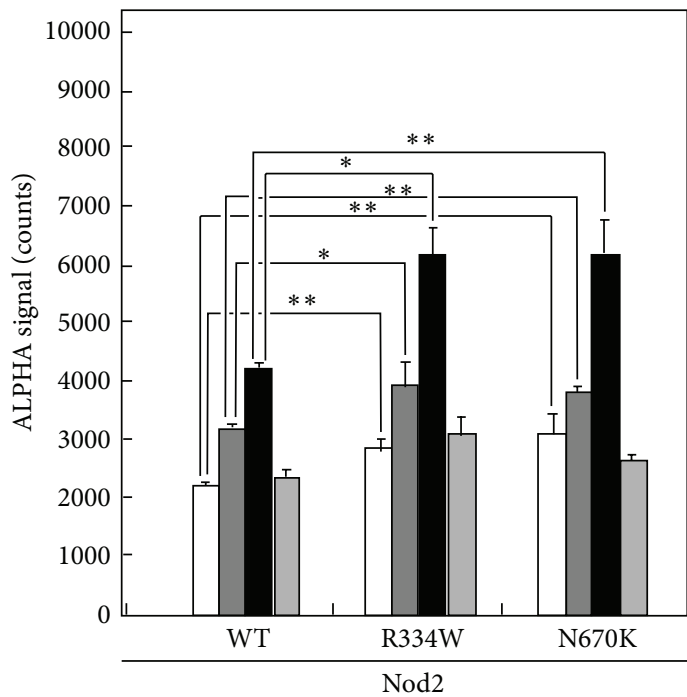

MDP-D-isomer $(13.33 \mathrm{mg} / \mathrm{mL}) \square \operatorname{MDP}(5.33 \mathrm{mg} / \mathrm{mL})$ $\operatorname{MDP}(13.33 \mathrm{mg} / \mathrm{mL})$

(c)

(d)

FIGURE 2: Construction of Nod2-nodosome containing the BS/EOS-associated mutation in a cell-free system. Synthetic protein-protein interactions were detected by pull-down assay and amplified luminescent proximity homogeneous assay (ALPHA). (a) Biotinylated-Nod2WT (Nod2-WT-Btn) and $1 \mu \mathrm{g}$ FLAG-tagged RICK-WT (FLAG-RICK-WT) lysed in $300 \mu \mathrm{L}$ NP-40 buffer were precipitated with $20 \mu \mathrm{L}$ streptavidin-conjugated agarose beads with or without MDP. The precipitations were subjected to SDS-PAGE and immunoblotting. Detection on the blotting membranes was performed using anti-FLAG mAb M2 or anti-Nod2 mAb. (b) A total of 100 ng of each protein indicated was incubated with $5 \mu \mathrm{g} / \mathrm{mL}$ anti-FLAG $\mathrm{mAb} \mathrm{M} 2,16.67 \mu \mathrm{g} / \mathrm{mL}$ protein-A-conjugated ALPHA acceptor beads, and $16.67 \mu \mathrm{g} / \mathrm{mL}$ streptavidinconjugated ALPHA donor beads for 24 hours, with or without $5.33 \mathrm{mg} / \mathrm{mL}$ MDP or $5 \mathrm{mg} / \mathrm{mL}$ N-Acetylmuramyl-D-Alanyl-D-Isoglutamine (MDP-D-isomer). Responses (counts) were measured using the EnSpire ${ }^{\mathrm{TM}}$ Multimode Plate Reader. (c) Activation of Nod2-nodosome in a cell-free system by MDP degradation components. Interactions between Nod2-WT-Btn and FLAG-RICK-WT were detected by ALPHA. A total of $100 \mathrm{ng}$ of Nod2-WT-Btn and FLAG-RICK-WT were incubated with $5 \mu \mathrm{g} / \mathrm{mL}$ anti-FLAG mAb M2, $16.67 \mu \mathrm{g} / \mathrm{mL}$ protein-A-conjugated ALPHA acceptor beads and $16.67 \mu \mathrm{g} / \mathrm{mL}$ streptavidin-conjugated ALPHA donor beads for 24 hours, without or with $5.33 \mathrm{mg} / \mathrm{mL} \mathrm{MDP}$, $5.33 \mathrm{mg} / \mathrm{mL}$ MDP (D-isomer), $5.33 \mathrm{mg} / \mathrm{mL} \mathrm{N}$-acetylglucosamine (GlcNAc), $5.33 \mathrm{mg} / \mathrm{mL} \mathrm{L}$-alanine (L-Ala), or $5.33 \mathrm{mg} / \mathrm{mL} \mathrm{D}$-isoglutamine (D-isoGlu). Responses (counts) were measured using the EnSpire Multimode Plate Reader. The results are representative of three independent experiments and given as means \pm standard deviation from triplicate wells. (d) A total of $100 \mathrm{ng}$ of Nod2-WT-Btn, or Nod2-R334W-Btn, or Nod2-N670K-Btn with FLAG-RICK-WT was incubated with $5 \mu \mathrm{g} / \mathrm{mL}$ anti-FLAG mAb M2, $16.67 \mu \mathrm{g} / \mathrm{mL}$ protein-A-conjugated ALPHA acceptor beads, and $16.67 \mu \mathrm{g} / \mathrm{mL}$ streptavidin-conjugated ALPHA donor beads for 24 hours, with or without $5.33 \mathrm{mg} / \mathrm{mL} \mathrm{or} 13.33 \mathrm{mg} / \mathrm{mL} \mathrm{MDP}$ or $13.33 \mathrm{mg} / \mathrm{mL}$ MDP-D-isomer. The results are representative of three independent experiments and given as means \pm standard deviation from triplicate wells. CARD, caspase recruitment domain; MDP, muramyl dipeptide; WT, wild-type; P, precipitation; WB, western blot. ${ }^{*} p$ value $<0.05$ and ${ }^{* *} p$ value $<0.01$ were considered statistically significant using Student's $t$-test. 
in a cell-free system with or without BS/EOS-associated mutations, we assessed the interaction between FLAG-RICKWT and Nod2-WT-Btn, Nod2-R334W-Btn, or Nod2-N670K$\mathrm{Btn}$, in a cell-free system. The ALPHA signal between FLAGRICK-WT and Nod2-WT-Btn increased upon incubation with $5.33 \mathrm{mg} / \mathrm{mL}$ and $13.33 \mathrm{mg} / \mathrm{mL}$ MDP, in a dose-dependent manner (Figure 2(d)). The baseline ALPHA signal between the BS/EOS-associated Nod2 mutants, such as Nod2-R334WBtn and Nod2-N670K-Btn, and FLAG-RICK-WT with no stimulation was higher than that between Nod2-WT-Btn and FLAG-RICK-WT $(p<0.01, p<0.01$, resp.) (Figure $2(\mathrm{~d})$, left columns). Upon incubation with $5.33 \mathrm{mg} / \mathrm{mL}$, the ALPHA signal between Nod2-WT-Btn and FLAG-RICK$\mathrm{WT}$ increased in a dose-dependent manner, and signals between Nod2-R334W-Btn or Nod2-N670K-Btn and FLAGRICK-WT were more significantly increased $(p<0.05$, $p<0.01$, resp.) (Figure 2(d), center columns). Upon incubation with $13.33 \mathrm{mg} / \mathrm{mL}$ MDP, the ALPHA signal between Nod2-WT-Btn and FLAG-RICK-WT increased in a dosedependent manner, and signals between Nod2-R334W-Btn or Nod2-N670K-Btn and FLAG-RICK-WT were more significantly increased ( $p<0.05, p<0.01$, resp.) (Figure 2(d), right columns).

\section{Discussion}

Autoinflammatory syndromes are known to stem from aberrant innate immune complex disorders, some of which are known to be due to the excessive innate immune activation by mutations of the pathogen-recognizing receptors [17]. $\mathrm{BS} / \mathrm{EOS}$ is one of the autoinflammatory syndromes, which is characterized by systemic chronic granulomatous inflammation, resulting in nonnecrotizing granuloma of the skin, eyes, and joints [12]. The susceptibility gene product of BS/EOS has been identified to be Nod2/CARD15 [11, 18].

In the present study, we developed Nod2-nodosomes containing wild-type and the BS/EOS-associated mutants R334W and N670K in a cell-free system, assessed by ALPHA. In general, recombinant protein synthesis has been used for bacterial or viral systems, such as Escherichia coli, for structural and functional studies. However, synthetic proteins sometimes are unable to be investigated, because many proteins are expressed in an insoluble form [19]. Therefore, we employed the wheat germ cell-free protein synthesis system, and Nod2-WT-Btn, Nod2-R334W-Btn, Nod2-N670K-Btn, Nod2-CARDs, FLAG-RICK-WT, and FLAG-RICK-CARD were successfully synthesized (Figure 1). To date, we reported the generation of AIM2-inflammasome in a cell-free system, which is another intracellular pattern recognition receptor with adaptor ASC oligomerization, which was suitable for wheat germ cell-free protein synthesis [20]. These results suggested that the wheat germ cell-free protein synthesis system has an advantage for reconstituting intracellular pattern recognition receptor complexes for resolving innate immune complex-mediated autoinflammatory diseases.

First, we confirmed whether the Nod2-nodosome could recognize the bacterial cell-wall component MDP, which is a previously reported Nod2-ligand $[6,7]$. As shown in
Figure 2(a), Nod2-WT-Btn was coprecipitated with FLAGRICK-WT when incubated with MDP, but not without MDP in pull-down assay (Figure 2(a)). That was consistent with ALPHA in Figure 2(b). As shown in Figure 2(b), the ALPHA signal of interaction between Nod2-WT-Btn and FLAG-RICK-WT was increased upon incubation with MDP, but not without a ligand or with the MDP-D-isomer or MDP-degraded components (Figures 2(b) and 2(c)). These results indicated that Nod2-nodosome in a cell-free system is capable of detecting for its specific ligand, MDP.

Next, we tested whether the BS/EOS-associated Nod2 mutations induced increased activity. As shown in Figure 2(d), the ALPHA signal of interaction between FLAGRICK-WT and Nod2-R334W-Btn or Nod2-N670K-Btn was much higher than when with Nod2-WT-Btn, with or without MDP stimulation (Figure 2(d)). These results are consistent with previous studies on the clinical manifestations of BS/EOS [21], suggesting that Nod2-nodosome in a cell-free system may suitably reflect the characteristics of endogenous Nod2-nodosome.

It is noted that there are some limitations of nodosome in a cell-free system. Only the initial event of Nod2-RICK interaction is detected. Upstream, downstream, and regulatory events in the activation of Nod 2 cannot be measured with the nodosome in a cell-free system, as presented.

In conclusion, we developed Nod2-nodosome in a cellfree system, assessed by ALPHA. Nod2-nodosomes containing the BS/EOS-associated mutations Nod2-R334W and Nod2-N670K were more sensitive to MDP than Nod2WT. Therefore, the Nod2-nodosomes in a cell-free system developed in the present study can be a useful tool for further investigation of pathogenesis of BS/EOS and discovery of its therapeutics.

\section{Disclosure}

The funders had no role in the study design, data collection, and analysis, decision to publish, or preparation of the paper.

\section{Competing Interests}

The authors have no competing interests.

\section{Authors' Contributions}

All authors were involved in drafting the paper. Junya Masumoto had full access to all data in the study and takes responsibility for the integrity of the data and accuracy of the data analysis. All authors approved the final version to be published. Tomoyuki Iwasaki and Junya Masumoto were responsible for study conception and design. Tomoyuki Iwasaki, Naoe Kaneko, Yuki Ito, Toshio Heike, Kiyoshi Migita, Kazunaga Agematsu, Atsushi Kawakami, Shinnosuke Morikawa, Sho Mokuda, Mie Kurata, and Junya Masumoto were responsible for the acquisition of data. Tomoyuki Iwasaki, Naoe Kaneko, Yuki Ito, Hiroyuki Takeda, Tatsuya Sawasaki, and Junya Masumoto were responsible for analysis and interpretation of data. 


\section{Acknowledgments}

This work was partially supported by the Practical Research Project for Rare/Intractable Diseases from Japan Agency for Medical Research and Development, AMED (Toshio Heike, Kiyoshi Migita, Kazunaga Agematsu, Atsushi Kawakami, and Junya Masumoto), and a Grant-in-Aid for Scientific Research (JSPS KAKENHI Grant nos. 26293232 and 26305024) and Platform for Drug Discovery, Informatics, and Structural Life Science from the Ministry of Education, Culture, Sports, Science, and Technology, Japan (Hiroyuki Takeda, Tatsuya Sawasaki, and Junya Masumoto).

\section{References}

[1] Y. Ogura, N. Inohara, A. Benito, F. F. Chen, S. Yamaoka, and G. Núñez, "Nod2, a Nod1/Apaf-1 Family member that is restricted to monocytes and activates NF- $\kappa \mathrm{B}$," Journal of Biological Chemistry, vol. 276, no. 7, pp. 4812-4818, 2001.

[2] Y. Ogura, D. K. Bonen, N. Inohara et al., "A frameshift mutation in NOD2 associated with susceptibility to Crohn's disease," Nature, vol. 411, no. 6837, pp. 603-606, 2001.

[3] J.-P. Hugot, M. Chamaillard, H. Zouali et al., "Association of NOD2 leucine-rich repeat variants with susceptibility to Crohn's disease," Nature, vol. 411, no. 6837, pp. 599-603, 2001.

[4] N. Inohara, L. Del Peso, T. Koseki, S. Chen, and G. Núñez, "RICK, a novel protein kinase containing a caspase recruitment domain, interacts with CLARP and regulates CD95-mediated apoptosis," The Journal of Biological Chemistry, vol. 273, no. 20, pp. 12296-12300, 1998.

[5] J. V. McCarthy, J. Ni, and V. M. Dixit, "RIP2 is a novel NF$\kappa \mathrm{B}$-activating and cell death-inducing kinase," The Journal of Biological Chemistry, vol. 273, no. 27, pp. 16968-16975, 1998.

[6] N. Inohara, Y. Ogura, A. Fontalba et al., "Host recognition of bacterial muramyl dipeptide mediated through NOD2: implications for Crohn's disease," Journal of Biological Chemistry, vol. 278, no. 8, pp. 5509-5512, 2003.

[7] S. E. Girardin, I. G. Boneca, J. Viala et al., "Nod2 is a general sensor of peptidoglycan through muramyl dipeptide (MDP) detection," The Journal of Biological Chemistry, vol. 278, no. 11, pp. 8869-8872, 2003.

[8] M. Sugimoto, R. N. Germain, L. Chedid, and B. Benacerraf, "Enhancement of carrier-specific helper $\mathrm{T}$ cell function by the synthetic adjuvant, $\mathrm{N}$-acetyl muramyl-L-alanyl-Disoglutamine (MDP)," Journal of Immunology, vol. 120, no. 3, pp. 980-982, 1978.

[9] M. Chamaillard, D. Philpott, S. E. Girardin et al., "Geneenvironment interaction modulated by allelic heterogeneity in inflammatory diseases," Proceedings of the National Academy of Sciences of the United States of America, vol. 100, no. 6, pp. 34553460, 2003.

[10] T. Tanabe, M. Chamaillard, Y. Ogura et al., "Regulatory regions and critical residues of NOD2 involved in muramyl dipeptide recognition," EMBO Journal, vol. 23, no. 7, pp. 1587-1597, 2004.

[11] N. Kanazawa, I. Okafuji, N. Kambe et al., "Early-onset sarcoidosis and CARD15 mutations with constitutive nuclear factor$\kappa \mathrm{B}$ activation: common genetic etiology with Blau syndrome," Blood, vol. 105, no. 3, pp. 1195-1197, 2005.

[12] E. B. Blau, "Familial granulomatous arthritis, iritis, and rash," The Journal of Pediatrics, vol. 107, no. 5, pp. 689-693, 1985.
[13] Q. Yao, "Nucleotide-binding oligomerization domain containing 2: structure, function, and diseases," Seminars in Arthritis and Rheumatism, vol. 43, no. 1, pp. 125-130, 2013.

[14] M. Shen, R. Moran, K. J. Tomecki, and Q. Yao, "Granulomatous disease associated with NOD2 sequence variants and familial camptodactyly: an intermediate form of NOD2-associated diseases?" Seminars in Arthritis and Rheumatism, vol. 45, no. 3, pp. 357-360, 2015.

[15] A. Biswas, Y.-J. Liu, L. Hao et al., "Induction and rescue of Nod2-dependent Th1-driven granulomatous inflammation of the ileum," Proceedings of the National Academy of Sciences of the United States of America, vol. 107, no. 33, pp. 14739-14744, 2010.

[16] I. Tattoli, L. H. Travassos, L. A. Carneiro, J. G. Magalhaes, and S. E. Girardin, "The nodosome: Nod1 and Nod2 control bacterial infections and inflammation," Seminars in Immunopathology, vol. 29, no. 3, pp. 289-301, 2007.

[17] M. J. Ombrello and D. L. Kastner, "Autoinflammation in 2010: expanding clinical spectrum and broadening therapeutic horizons," Nature Reviews Rheumatology, vol. 7, no. 2, pp. 82-84, 2011.

[18] C. Miceli-Richard, S. Lesage, M. Rybojad et al., "CARD15 mutations in Blau syndrome," Nature Genetics, vol. 29, no. 1, pp. 19-20, 2001.

[19] N. J. Saez and R. Vincentelli, "High-throughput expression screening and purification of recombinant proteins in E. coli," Methods in Molecular Biology, vol. 1091, pp. 33-53, 2014.

[20] N. Kaneko, Y. Ito, T. Iwasaki et al., "Reconstituted AIM2 inflammasome in cell-free system," Journal of Immunological Methods, vol. 426, pp. 76-81, 2015.

[21] I. Okafuji, R. Nishikomori, N. Kanazawa et al., "Role of the NOD2 genotype in the clinical phenotype of Blau syndrome and early-onset sarcoidosis," Arthritis and Rheumatism, vol. 60, no. 1, pp. 242-250, 2009. 


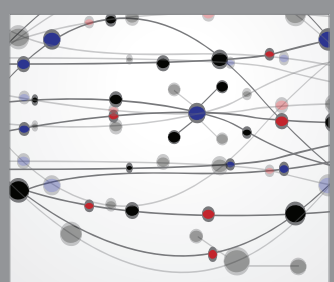

The Scientific World Journal
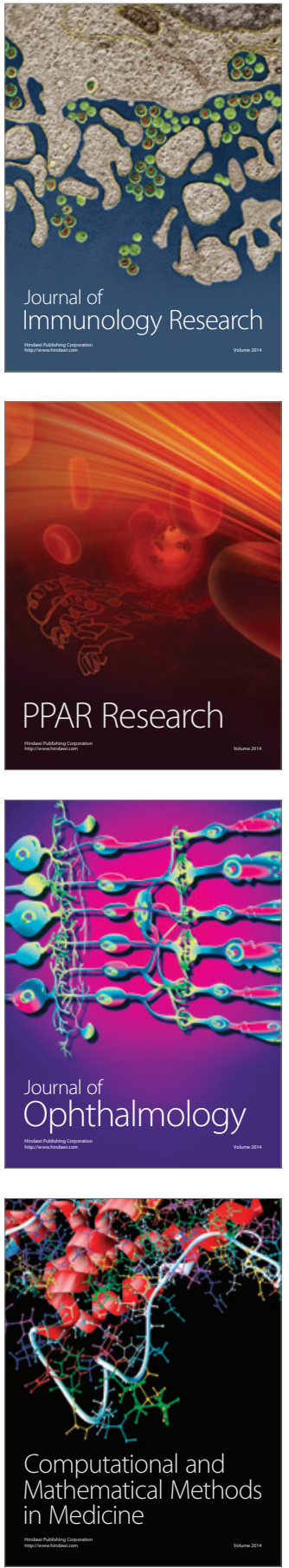

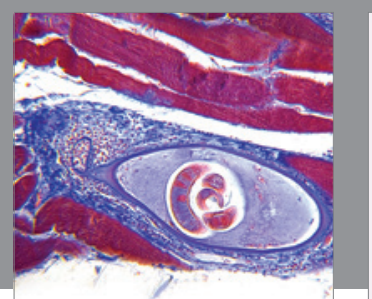

Gastroenterology Research and Practice

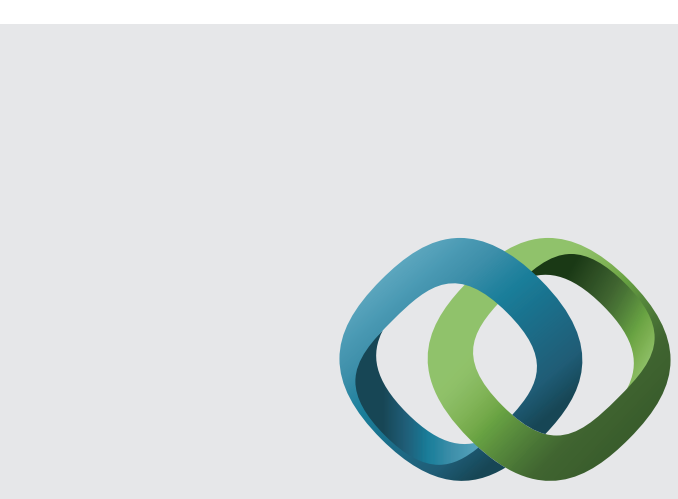

\section{Hindawi}

Submit your manuscripts at

http://www.hindawi.com
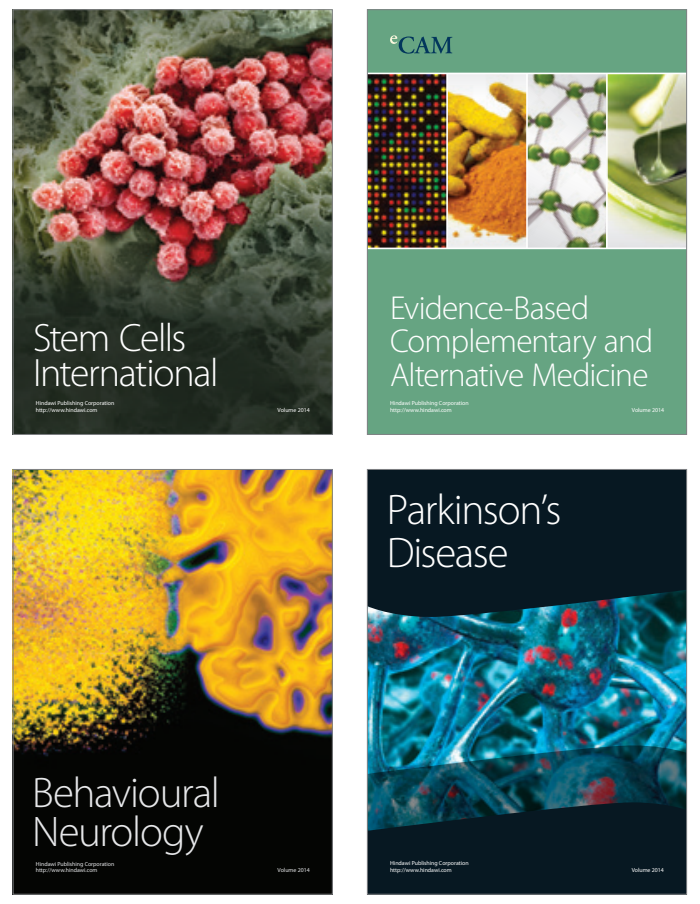
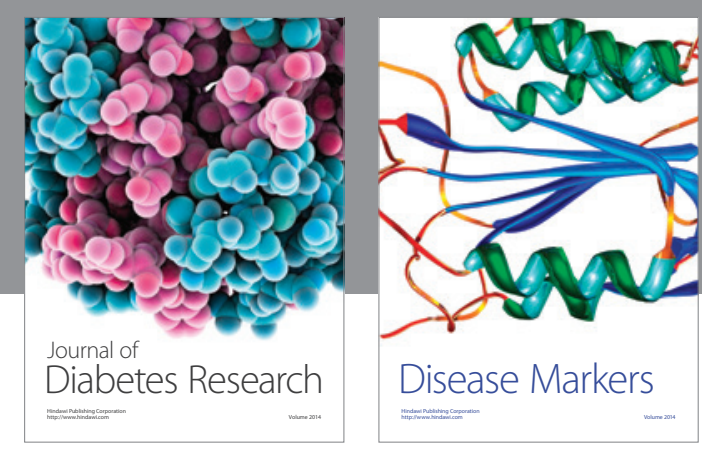

Disease Markers
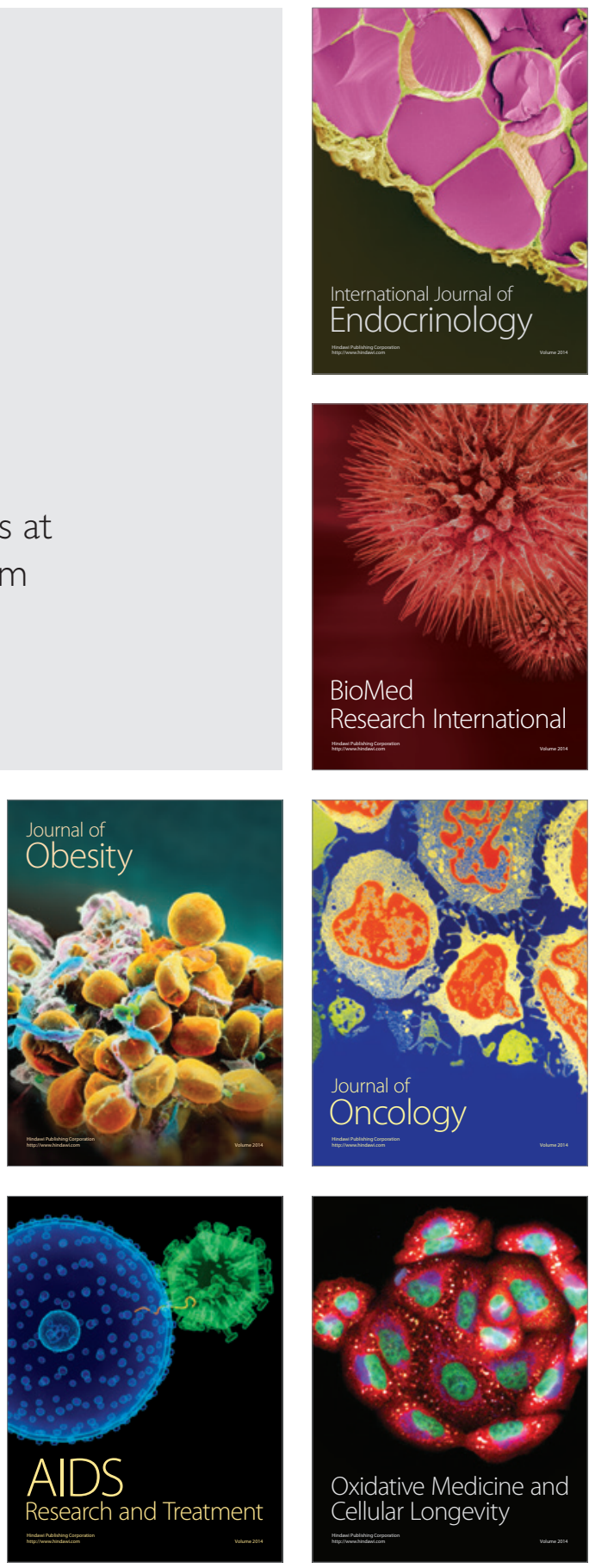